\title{
Effects of repairing ventricular septal defects with right vertical infra-axillary mini-incision on lung function and postoperative analgosedation
}

\author{
CHUNXIANG LI, XIAOLEI GONG, JIHONG HUANG, LIMIN ZHU, WEI DONG, \\ MINGJIE ZHANG, YUJIE LIU and ZHUOMING XU \\ Department of Cardiac Intensive Care Unit, Shanghai Children's Medical Center, \\ Shanghai Jiaotong University School of Medicine, Shanghai 200127, P.R. China
}

Received June 30, 2020; Accepted January 6, 2021

DOI: $10.3892 /$ etm.2021.9733

\begin{abstract}
The present study aimed to assess the effects of repairing ventricular septal defects (VSDs) with right vertical infra-axillary mini-incision (RVAI). A total of 116 patients with VSDs were prospectively enrolled and underwent cardiac surgery between June 2017 and December 2018 at the cardiac intensive care unit of Shanghai Children's Medical Center (Shanghai, China). Of these, 58 patients underwent the RVAI procedure and 58 patients matched 1:1 underwent the standard median sternotomy incision (MSI) procedure and were designated as the control group. The demographic data and clinical outcomes intra- and postoperatively were compared. A bedside lung ultrasound was performed to evaluate the degree of lung injury and the number of B-lines was quantified and compared between the two groups. The sedation and analgesia levels were also assessed after the operation. No significant difference was identified between the two groups regarding the overall cardiopulmonary bypass or aortic cross-clamp time. All patients were extubated within $8 \mathrm{~h}$. The RVAI group had shorter incision lengths (median, $4.6 \mathrm{~cm}$ ) and less drainage (median, $15 \mathrm{ml}$ ) than the MSI group. Furthermore, compared to the MSI group, the RVAI group had a significantly higher number of B-lines in the right lung regions immediately after surgery and at $12 \mathrm{~h}$ postsurgery (24.1 and $5.2 \%$, respectively) but eventually exhibited no differences at 24 and 36 h postsurgery; by contrast, there were no differences in the left lung regions. The bedside bispectral index score and the Face, Legs, Activity, Cry, Consolability scale score exhibited no significant differences after the operation. In conclusion, the RVAI
\end{abstract}

Correspondence to: Dr Zhuoming Xu, Department of Cardiac Intensive Care Unit, Shanghai Children's Medical Center, Shanghai Jiaotong University School of Medicine, 1,678 Dongfang Road, Shanghai 200127, P.R. China

E-mail: zmxyfb2018@163.com

Key words: right vertical infra-axillary mini-incision, median sternotomy incision, ultrasound B-lines, analgosedation procedure appears to be a safe alternative for repairing VSDs in addition to satisfactory cosmetic results and the incision does not interfere with postoperative analgosedation.

\section{Introduction}

Median sternotomy incision (MSI) is considered the usual approach for treating congenital heart defects (CHDs). For simple and common CHDs, such as atrial and ventricular septal defects (VSDs), undesirable cosmetic results and sternotomy-related complications may limit the number of surgeons who choose to perform median sternotomy procedures (1). Several minimally invasive cardiac surgery techniques have been increasingly explored to overcome these disadvantages (2-6). Over the past few years, right vertical infra-axillary mini-incision (RVAI) has been utilized to obtain reliable cosmetic and functional results in patients with CHDs $(7,8)$. In addition, Dave et al $(7)$ reported that repairs for various congenital defects were similar in quality to standard surgical approaches. However, the patient was placed in a left lateral position and the lung was kept away from the heart during the operation, which may cause detriments to the lung. Irritation to the intercostal nerves during minimally invasive procedures (9) may make postoperative sedation and analgesia difficult. To the best of our knowledge, no similar studies have been reported.

In the present study, MSI and RVAI were compared in terms of their ability to close VSDs, the demographics and clinical outcomes of patients were compared and it was investigated whether this novel technique affects postoperative lung function or postoperative pain.

\section{Materials and methods}

Study design. The present study was a prospective cohort study performed at the cardiac intensive care unit (CICU) of Shanghai Children's Medical Center (Shanghai, China) and included pediatric patients with VSDs who underwent corrective operations between June 2017 and December 2018. The present study was approved by the Ethics Committee of Shanghai Jiaotong University School of Medicine (Shanghai, 
China; approval no. SCMCIRB-K2017067) and written informed consent was obtained from the patients' parents.

\section{Operative technique}

RVAI approach. After inducing general anesthesia, the patient was placed in the left lateral decubitus position with the right side elevated $45-60^{\circ}$ and the right arm was wrapped and suspended over the head. A vertical incision was made on the right midaxillary line of the skin from the second to the fifth ribs and the surgical route generally went through the fourth intercostal space. After the defect was closed, a pericardial drain was inserted. The subcutaneous tissue and skin were sutured in layers (7).

MSI approach. With the patient in the supine position, the heart was exposed through a classic midline sternotomy. The sternum was divided wholly and filled with wax to stop the bleeding. Through aortic, inferior and superior vena caval cannulations, a mildly hypothermic cardiopulmonary bypass (CPB) was established. After the defect was closed, a pericardial drain was inserted and the divided sternum was closed with non-absorbable sutures. The subcutaneous tissue and skin were sutured in layers.

Patient selection. All patients included in the present study had VSDs diagnosed by physical examinations, chest X-ray, electrocardiography, transthoracic color Doppler echocardiography and CT. A flow chart of the selection of the study population is provided in Fig. 1. In total, 58 patients underwent surgery with the RVAI technique between June 2017 and December 2018 (RVAI group). To balance the major prognostic factors, a 1:1 matched-pairs case-control methodology was designed and the control group underwent the standard MSI procedure (MSI group). The patients of each group were similarly matched in terms of corresponding heart defects, year of surgery, body weight (the difference was $\leq 2 \mathrm{~kg}$ ) and patching (Table I).

The intraoperative variables (aortic clamp time and cardiopulmonary bypass time) were recorded in both groups. Furthermore, the extubation time was evaluated with early extubation defined as mechanical ventilation that lasted for $<8 \mathrm{~h}$ postoperatively. The incision length and drainage volume were also calculated (Table I).

Lung ultrasonography examination. After the patients returned to the CICU, the right and left lungs were routinely examined by ultrasound (every $12 \mathrm{~h}$ ) to evaluate the injury with B-lines (10) immediately after surgery $\left(\mathrm{H}_{0}\right)$ at $12 \mathrm{~h}$ postoperatively $\left(\mathrm{H}_{12}\right)$, as well as $\mathrm{H}_{24}$ and $\mathrm{H}_{36}$. The number of $\mathrm{B}$-lines was summed up to generate quantitative or semiquantitative B-line scores. The lung ultrasound probe requires a high-frequency linear array of $>7.5 \mathrm{MHz}$. Lung ultrasonography (LUS) examinations were performed according to the methodology described by Copetti and Cattarossi (11). The patients were positioned in a supine, lateral or prone position. The lung field was divided into three areas by the midclavicular line and anterior axillary line. The regions of the bilateral lung were scanned with the probe, which was held vertical or parallel to the ribs. Each region was scanned in the longitudinal and transverse planes and in the medial-lateral and cranial-caudal directions, respectively.

Sedation and analgesia assessment. The patients returned to the CICU with a bispectral index score (BIS) monitor (BIS

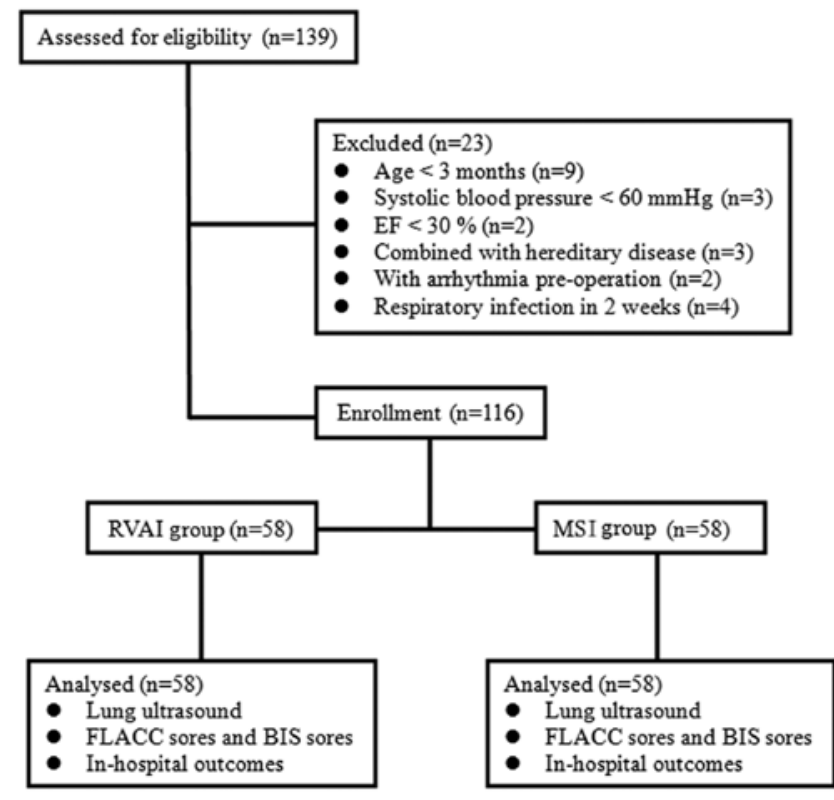

Figure 1. Flow chart depicting the selection of the study population. RVAI, right vertical infra-axillary mini-incision; MSI, median sternotomy incision; FLACC, Face, Legs, Activity, Cry, Consolability scale; BIS, bispectral index score; EF, ejection fraction.

Vista model; Aspect Medical Systems) until the day they left the CICU. The BIS monitor was able to assess the anesthetic state of the central nervous system. The BIS scores ranged from 0 to 100 ( 0 , coma; 40-60, general anesthesia; 60-90, sedated; 100, awake) and reflect the level of sedation (12). The BIS scale was used to assess the sedation level at the CICU every $4 \mathrm{~h}$ after the operations $\left(\mathrm{H}_{0}, \mathrm{H}_{4}, \mathrm{H}_{8}, \mathrm{H}_{12} ;\right)$. The Face, Legs, Activity, Cry and Consolability (FLACC) scale was used to assess the analgesia level at the bedside after the patient was weaned from ventilation $\left(\mathrm{H}_{8}, \mathrm{H}_{12}\right)$. The FLACC scale consists of 5 categories (face, legs, activity, cry and consolability), each with a score between 0 and 2 , with 10 being the full score; scores of 0 to 3,4 to 7 and 8 to 10 represent no/light pain, moderate pain and acute pain, respectively. The FLACC scale is reliable and valid for the assessment of systematic pain in children (13). There were no differences in the application of anesthesia, sedation and analgesia medication between the two groups. Both imidazole $2 \mu \mathrm{g} / \mathrm{kg} / \mathrm{min}$ and dexmedetomidine $0.5 \mu \mathrm{g} / \mathrm{kg} / \mathrm{h}$ were provided for $16 \mathrm{~h}$.

Statistical analysis. Statistical analysis was performed using SPSS version 19.0 (IBM Corp.). Data following a normal distribution are expressed as the median (range) or as the mean \pm standard deviation. Variables were compared between the RVAI and MSI groups using a Mann-Whitney U test for data with equal proportions or Fisher's exact test where numbers were small with results presented as $\mathrm{n}(\%)$. The statistical tests were two-sided. All the analyses were performed on an intent-to-treat basis and a two-sided $\mathrm{P}<0.05$ was considered to indicate statistical significance.

\section{Results}

Operation procedures and enrolling groups. All procedures were successfully performed and none of the procedures 
Table I. Patient characteristics of the study groups.

\begin{tabular}{|c|c|c|c|}
\hline Variable & RVAI group $(n=58)$ & MSI group (n=58) & P-value \\
\hline Age (months) & $14.5(6-31)$ & $14(5.5-30)$ & 0.69 \\
\hline Male sex & $24(41.4 \%)$ & $25(43.1)$ & 0.83 \\
\hline Body weight (kg) & $10(6.8-15.7)$ & $9.8(6.5-15)$ & 0.73 \\
\hline Aortic clamp time (min) & $19(4-39)$ & $18(4-38)$ & 0.63 \\
\hline CPB time (min) & $40(14-73)$ & $39(13-70)$ & 0.67 \\
\hline Mechanical ventilation (h) & $4.30(3.83-7.25)$ & $4.25(3.75-7.72)$ & 0.75 \\
\hline Incision length (cm) & $4.6(4-6)$ & $6.1(4.8-8)$ & 0.04 \\
\hline Drainage volume (ml) & $15(5-80)$ & $45(10-125)$ & 0.03 \\
\hline Perimembranous VSDs n (\%) & $55(94.8)$ & $54(93.1)$ & 0.63 \\
\hline Combined with ASD n (\%) & $26(44.8)$ & $27(46.6)$ & 0.71 \\
\hline Combined with TR n (\%) & $20(34.5)$ & $19(32.8)$ & 0.82 \\
\hline VSD size & $8.1 \pm 3.5$ & $7.9 \pm 3.7$ & 0.61 \\
\hline EF after the operation & $52.5 \pm 13.2$ & $53.1 \pm 12.8$ & 0.45 \\
\hline Re-operation or re-CPB n (\%) & $0(0)$ & $0(0)$ & 0.73 \\
\hline CPAP support n (\%) & $2(3.4)$ & $0(5.1)$ & 0.77 \\
\hline ECMO support n (\%) & $0(0)$ & $0(0)$ & 0.65 \\
\hline Family hereditary disease n (\%) & $0(0)$ & $0(0)$ & 0.34 \\
\hline Premature delivery n (\%) & $3(5.2)$ & $0(6.9)$ & 0.55 \\
\hline Inotropic score & $5.5 \pm 2.5$ & $5.5 \pm 3.0$ & 0.78 \\
\hline Re-thoracotomy n (\%) & $1(1.7)$ & $1(1.7)$ & 0.43 \\
\hline
\end{tabular}

Values are expressed as the median (range), the mean \pm standard deviation or $\mathrm{n}(\%)$. RVAI, right vertical infra-axillary mini-incision; MSI, median sternotomy incision; VSD, ventricular septal defect; ASD, atrial septal defect; EF, ejection fraction; CPB, cardiopulmonary bypass; TR, tricuspid regurgitation; CPAP, continuous positive airway pressure; ECMO, extracorporeal membrane oxygenation.

A

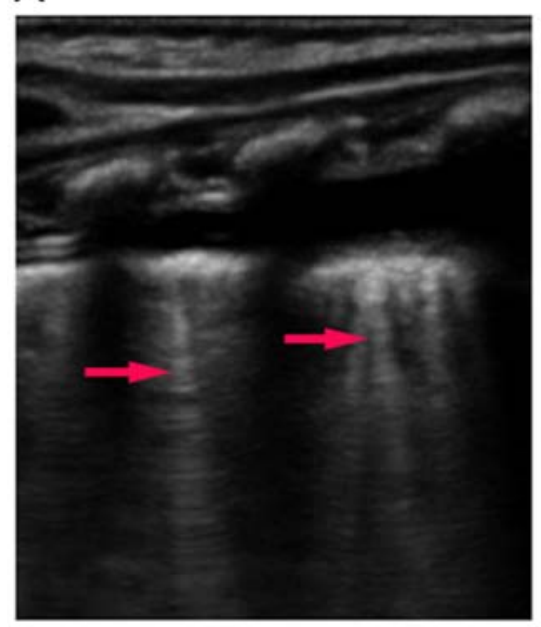

B

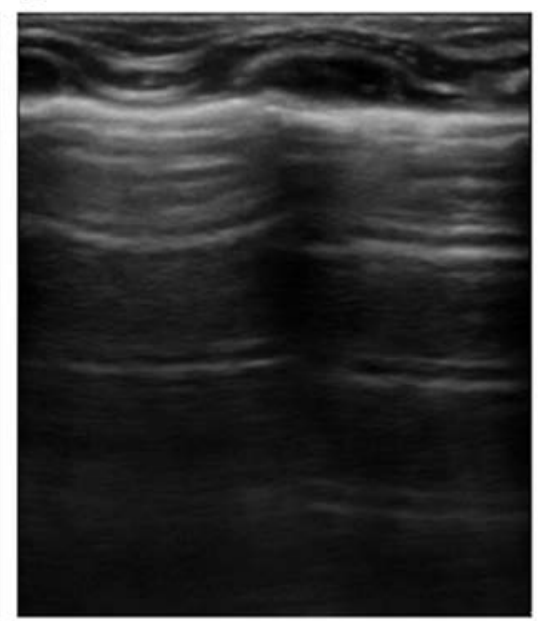

Figure 2. Ultrasound images of lung after surgery. (A) Ultrasound images of B-lines (indicated by red arrows) arising from the pleural line and spreading up to the edge of the screen. (B) Ultrasonography image exhibiting signs of a relatively normal lung.

was required to be converted to another approach. None of the patients required reoperation for bleeding. A total of 116 pediatric patients with VSDs were admitted to the CICU following cardiac surgery. Among these patients, 58 underwent the RVAI technique, while 58 matched patients who underwent the standard MSI procedure were designated as the control group.
Characteristics of the Patients. The baseline characteristics of the 116 patients are presented in Table I. The age (5.5-31 months), sex (49 male, 42.2\%), body weight, aortic clamp time, CPB time and duration of mechanical ventilation were not significantly different between the RVAI group and the MSI control group $(\mathrm{P}>0.05)$. All patients were extubated within 8 h. The RVAI group had shorter incision lengths 
Table II. Positive detection rate of B-lines and subcutaneous emphysema by lung ultrasound $\left(\mathrm{H}_{0}, \mathrm{H}_{12}, \mathrm{H}_{24}\right.$ and $\left.\mathrm{H}_{36}\right)$.

\begin{tabular}{|c|c|c|c|}
\hline $\begin{array}{l}\text { B-line location/ } \\
\text { time-point }\end{array}$ & $\begin{array}{l}\text { RVAI group } \\
\quad(n=58)\end{array}$ & $\begin{array}{l}\text { MSI group } \\
\quad(n=58)\end{array}$ & P-value \\
\hline \multicolumn{4}{|l|}{ Right lung } \\
\hline $\mathrm{H}_{0}$ & $14(24.1)$ & $3(5.2)$ & 0.001 \\
\hline $\mathrm{H}_{12}$ & $10(17.2)$ & $2(3.4)$ & 0.002 \\
\hline $\mathrm{H}_{24}$ & $5 \quad(8.6)$ & $3(5.2)$ & 0.285 \\
\hline $\mathrm{H}_{36}$ & $1 \quad(1.7)$ & $2(3.4)$ & 0.655 \\
\hline \multicolumn{4}{|l|}{ Left lung } \\
\hline $\mathrm{H}_{0}$ & $2(3.4)$ & $3(5.2)$ & 0.480 \\
\hline $\mathrm{H}_{12}$ & $2 \quad(3.4)$ & $3(5.2)$ & 0.452 \\
\hline $\mathrm{H}_{24}$ & 1 (1.7) & $2(3.4)$ & 0.385 \\
\hline $\mathrm{H}_{36}$ & 1 (1.7) & $2(3.4)$ & 0.385 \\
\hline
\end{tabular}

Values are expressed as $\mathrm{n}(\%)$. RVAI, right vertical infra-axillary mini-incision; MSI, median sternotomy incision; $\mathrm{H}_{0}$, immediately after surgery; $\mathrm{H}_{8}, 8 \mathrm{~h}$ postoperatively.

Table III. FLACC scores $\left(\mathrm{H}_{8}, \mathrm{H}_{12}\right)$ and BIS scores of the patients measured every $4 \mathrm{~h}\left(\mathrm{H}_{0}, \mathrm{H}_{4}, \mathrm{H}_{8}, \mathrm{H}_{12}\right)$ at the cardiac intensive care unit after the operation.

\begin{tabular}{lccc}
\hline $\begin{array}{l}\text { Variable/ } \\
\text { time-point }\end{array}$ & $\begin{array}{c}\text { RVAI group } \\
(\mathrm{n}=58)\end{array}$ & $\begin{array}{c}\text { MSI group } \\
(\mathrm{n}=58)\end{array}$ & P-value \\
\hline $\mathrm{BIS}$ & & & \\
$\mathrm{H}_{0}$ & $61.2 \pm 7.56$ & $61.3 \pm 6.71$ & 0.92 \\
$\mathrm{H}_{4}$ & $72.2 \pm 4.23$ & $72.3 \pm 3.99$ & 0.96 \\
$\mathrm{H}_{8}$ & $79.8 \pm 4.78$ & $79.9 \pm 4.61$ & 0.95 \\
$\mathrm{H}_{12}$ & $75.8 \pm 6.78$ & $74.9 \pm 7.61$ & 0.85 \\
$\mathrm{FLACC}$ & & & \\
$\mathrm{H}_{8}$ & $5.86 \pm 0.86$ & $5.57 \pm 0.85$ & 0.26 \\
$\mathrm{H}_{12}$ & $5.91 \pm 0.67$ & $5.89 \pm 0.75$ & 0.45 \\
\hline
\end{tabular}

FLACC, Face, Legs, Activity, Cry, Consolability scale; BIS, bispectral index score; RVAI, right vertical infra-axillary mini-incision; MSI, median sternotomy incision; $\mathrm{H}_{0}$, immediately after surgery; $\mathrm{H}_{8}$, $8 \mathrm{~h}$ postoperatively.

(median, $4.6 \mathrm{~cm}$, range 4-6 cm; P>0.05; and Table I) and less drainage (median, $15 \mathrm{ml}$; range, $5-80 \mathrm{ml}$ ) than the MSI group.

Evaluation postoperative lung impairment and analgosedation. In the right lung, the patients in the RVAI group exhibited significantly more B-lines on LUS at $\mathrm{H}_{0}$ and $\mathrm{H}_{12}$ than the patients in the MSI group $(\mathrm{P}<0.05)$ and the positive detection $(>5 \mathrm{~B}$-lines) rate of B-lines was higher in the RVAI group than in the MSI group ( 24.1 vs. $5.2 \%$ at $\mathrm{H}_{0}, 17.2$ vs. $3.4 \%$ at $\mathrm{H}_{12}$ ). These results have not been previously investigated in the left lung and the number of B-lines was not different between groups at $\mathrm{H}_{24}$ and $\mathrm{H}_{36}(\mathrm{P}>0.05$; Fig. 2 and Table II). Patients in both groups exhibited almost the same BIS scores after each 4-h interval ( $\mathrm{P}>0.05$; Table III) and FLACC scores at $\mathrm{H}_{8}$ and $\mathrm{H}_{12}(\mathrm{P}>0.05$; Table III).

\section{Discussion}

In recent years, a minimally invasive cardiac surgery technique (2-6) has been increasingly explored to avoid mid-sternotomy scars and deformities and has achieved favorable cosmetic results and excellent repairs. RVAI is an emerging strategy for treating congenital heart disease that uses techniques that aim to minimize incisions and has led to the combination of reliable functional and cosmetic results $(7,8)$. In the present study, 116 patients with VSDs were enrolled between June 2017 and December 2018. MSI and RVAI were compared for their ability to close VSDs and the effects were evaluated intraoperatively and postoperatively.

Various studies have reported on the feasibility of performing the right axillary incision procedure to repair CHDs (3,7). Dave et al (7) reported that right axillary incision may be used to repair various congenital defects with a quality similar to that of standard surgical approaches while obtaining a superior cosmetic result compared to conventional incisions. Yan et al (3) also investigated whether RVAI was a safe procedure and determined that RVAI may be performed with excellent cosmetic and clinical outcomes. Similarly, in the present study, no difference in the number of transfusions was identified between the groups, and the MSI group had similar cardiopulmonary bypass and cross-clamp times. Cosmetic results are important for patients who undergo surgery to close simple CHDs, and the short incision length and location of the RVAI, the remaining scar of which is hidden by the resting arm, results in satisfactory cosmetic effects (3). Furthermore, the RVAI group had less drainage from the incision than the MSI group; this result may be attributed to the sternum not having been split, which results in less bleeding. Pectus deformities have been reported after standard MSI surgeries to treat CHDs (14). In the present study, no thorax asymmetry was detected in the RVAI group 6 months after operation.

In recent decades, ultrasound has become an important method for diagnostic examinations and monitoring of lung diseases; ultrasound has become an emerging diagnostic tool for diagnosing extravascular lung water with high sensitivity and specificity $(15,16)$. The B-lines of ultrasound images are signs of interstitial inflammation and congestion, which is critically important information in various common clinical situations $(17,18)$. In the present study, B-lines were applied to nonradioactively and conveniently evaluate lung impairment in the patients after surgery. Of note, in the right lung, patients in the RVAI group exhibited significantly more B-lines on LUS at $\mathrm{H}_{0}$ and $\mathrm{H}_{12}$ than those in the MSI group, but the results did not differ in the left lung. The number of B-lines was not different between the two groups at $\mathrm{H}_{24}$ and $\mathrm{H}_{36}$. These results indicated that the RVAI procedure may induce congestion or inflammatory reactions in the right lung within $12 \mathrm{~h}$ after the operation. However, all patients were extubated within $8 \mathrm{~h}$ after the operation and no pneumonia was diagnosed (data not shown). The present results implied that transient lung impairment from RVAI does not affect patient prognosis and all patients recovered rapidly within $24 \mathrm{~h}$.

The BIS score is objective and credible for the evaluation of analgosedation (12). In the present study, the level of postoperative pain was assessed with the BIS and FLACC scores to evaluate whether the RVAI procedure irritated the intercostal nerves 
and induced a higher intensity pain than the MSI procedure. A previous study reported that the minimally invasive procedure may cause intense pain (visual analog scale, 6.3) during the first three days after the operation (9). In the present study, the patients in the two groups had almost the same BIS score postoperatively after each 4-h interval. Furthermore, the FLACC scores after being weaned off ventilation were not significantly different between the RVAI group and the MSI control group. All of these results suggested that the RVAI procedure did not irritate the intercostal nerves to cause intense pain.

In conclusion, the RVAI procedure represents a safe alternative for fixing VSDs and leads to satisfactory cosmetic results; no thorax asymmetry was observed. Furthermore, the transient lung impairment of RVAI was rapidly recovered within $24 \mathrm{~h}$ and all patients were extubated within $8 \mathrm{~h}$ after the operation. The incision did not interfere with postoperative sedation and pain. To the best of our knowledge, the present study was the first comprehensive evaluation of RVAI to repair VSDs, reporting on the demographics and clinical outcomes of patients and investigating postoperative lung functions and postoperative pain. However, as it was only a single-center study, it the present results require to be confirmed by expanding the sample and performing multicenter trials.

\section{Acknowledgements}

The authors would like to thank Miss Liping Liu (Cardiac Intensive Care Unit, Shanghai Children's Medical Center, Shanghai Jiaotong University School of Medicine) for her cautious work and general supervision of the research group.

\section{Funding}

This work was supported by the National Natural Science Foundation of China (grant no. 81771934).

\section{Availability of data and materials}

The datasets used and/or analysed during the current study are available from the corresponding author on reasonable request.

\section{Authors' contributions}

CL, LZ and ZX designed the study. YL and MZ collected the data. JH, CL, YL, MZ and XG analyzed the data. CL, YL and $\mathrm{MZ}$ prepared the manuscript. CL, LZ and $\mathrm{JH}$ revised the manuscript. WD performed the operations. CL and ZX confirm the authenticity of all the raw data. All authors read and approved the final manuscript.

\section{Ethics approval and consent to participate}

The present study was approved by the Ethics Committee of Shanghai Jiaotong University School of Medicine (Shanghai, China) and written informed consent was obtained from the patients' parents.

\section{Patient consent for publication}

Not applicable.

\section{Competing interests}

The authors declare that they have no competing interests.

\section{References}

1. Luo H, Wang J, Qiao C, Zhang X, Zhang W and Song L: Evaluation of different minimally invasive techniques in the surgical treatment of atrial septal defect. J Thorac Cardiovasc Surg 148: 188-193, 2014.

2. Li G, Su J, Fan X, Li Z, Zhang J, Zhu Y, Liu A, Xu Y, Xu Y and Liu Y: Safety and efficacy of ventricular septal defect repair using a cosmetic shorter right lateral thoracotomy on infants weighing less than $5 \mathrm{~kg}$. Heart Lung Circ 24: 898-904, 2015.

3. Yan L, Zhou ZC, Li HP, Lin M, Wang HT, Zhao ZW, Gao QY and Ge JJ: Right vertical infra-axillary mini-incision for repair of simple congenital heart defects: A matched-pair analysis. Eur J Cardiothoracic Surg 43: 136-141, 2013.

4. Seipelt RG, Popov A, Danner B, Paul T, Tirilomis T, Schoendube FA and Ruschewski W: Minimally invasive partial inferior sternotomy for congenital heart defects in children. J Cardiovasc Surg (Torino) 51: 929-933, 2010.

5. Ma ZS, Yang CY, Dong MF, Wu SM and Wang LX: Totally thoracoscopic closure of ventricular septal defect without a robotically assisted surgical system: A summary of 119 cases. J Thorac Cardiovasc Surg 147: 863-867, 2014.

6. Xiao C, Gao C, Yang M, Wang G, Wu Y, Wang J, Wang R and Yao M: Totally robotic atrial septal defect closure: 7-year single-institution experience and follow-up. Interact Cardiovasc Thorac Surg 19: 933-937, 2014.

7. Dave HH, Comber M, Solinger T, Bettex D, Dodge-Khatami A and Prêtre R: Mid-term results of right axillary incision for the repair of a wide range of congenital cardiac defects. Eur J Cardiothorac Surg 35: 864-870, 2009.

8. Gil-Jaurena JM, Zabala JI, Conejo L, Cuenca V, Picazo B, Jiménez C, Castillo R, Ferreiros $M$, de Mora $M$ and Gutiérrez de Loma J: Minimally invasive pediatric cardiac surgery. Atrial septal defect closure through axillary and submammary approaches. Rev Esp Cardiol 64: 208-212, 2011.

9. Walther T,Falk V, Metz S, Diegeler A, Battellini R, Autschbach R and Mohr FW: Pain and quality of life after minimally invasive versus conventional cardiac surgery. Ann Thorac Surg 67: 1643-1647, 1999.

10. Picano E and Pellikka PA: Ultrasound of extravascular lung water: A new standard for pulmonary congestion. Eur Heart J 37: 2097-2104, 2016.

11. Copetti R and Cattarossi L: Ultrasound diagnosis of pneumonia in children. Radiol Med 113: 190-198, 2008 (In En, Italian).

12. Sadhasivam S, Ganesh A, Robison A, Kaye R and Watcha MF: Validation of the bispectral index monitor for measuring the depth of sedation in children. Anesth Analg 102: 383-388, 2006.

13. McGrath PJ, Walco GA, Turk DC, Dworkin RH, Brown MT, Davidson K, Eccleston C, Finley GA, Goldschneider K, Haverkos L, et al: Core outcome domains and measures for pediatric acute and chronic/recurrent pain clinical trials: PedIMMPACT recommendations. J Pain 9: 771-783, 2008.

14. Chen C, Li X, Zhang N, Yu J, Yan D, Xu C, Zeng Q and Li Z: Different Nuss procedures and risk management for pectus excavatum after surgery for congenital heart disease. J Pediatr Surg 53: 1964-1969, 2018.

15. Lichtenstein D, Mézière G, Biderman P, Gepner A and Barré O: The comet-tail artifact. An ultrasound sign of alveolar-interstitial syndrome. Am J Respir Crit Care Med 156: 1640-1646, 1997.

16. Gheorghiade M, Follath F, Ponikowski P, Barsuk JH, Blair JE, Cleland JG, Dickstein K, Drazner MH, Fonarow GC, Jaarsma T, et al: Assessing and grading congestion in acute heart failure: A scientific statement from the acute heart failure committee of the heart failure association of the European Society of Cardiology and endorsed by the European Society of Intensive Care Medicine. Eur J Heart Fail 12: 423-433, 2010.

17. Gargani L and Volpicelli G: How I do it: Lung ultrasound. Cardiovasc Ultrasound 12: 25, 2014.

18. Volpicelli G, Elbarbary M, Blaivas M,Lichtenstein DA, Mathis G, Kirkpatrick AW, Melniker L, Gargani L, Noble VE, Via G, et al: International evidence-based recommendations for point-of-care lung ultrasound. Intensive Care Med 38: 577-591, 2012. 